Article

\title{
Dissipative Ion-Acoustic Solitary Waves in Magnetized $\kappa$-Distributed Non-Maxwellian Plasmas
}

\author{
Sharmin Sultana ${ }^{1, *}$ and Ioannis Kourakis ${ }^{2}$ \\ 1 Department of Physics, Jahangirnagar University, Savar, Dhaka 1342, Bangladesh \\ 2 Department of Mathematics, College of Science and Engineering, Khalifa University of Science and \\ Technology, Abu Dhabi 127788, United Arab Emirates; ioanniskourakissci@gmail.com \\ * Correspondence: ssultana@juniv.edu
}

\section{check for}

updates

Citation: Sultana, S.; Kourakis, I. Dissipative Ion-Acoustic Solitary Waves in Magnetized $\kappa$-Distributed Non-Maxwellian Plasmas. Physics 2022, 4, 68-79. https://doi.org/ $10.3390 /$ physics 4010007

Received: 21 October 2021 Accepted: 3 January 2022

Published: 20 January 2022

Publisher's Note: MDPI stays neutral with regard to jurisdictional claims in published maps and institutional affiliations.

Copyright: (C) 2022 by the authors. Licensee MDPI, Basel, Switzerland. This article is an open access article distributed under the terms and conditions of the Creative Commons Attribution (CC BY) license (https:// creativecommons.org/licenses/by/ $4.0 /)$.

\begin{abstract}
The propagation of dissipative electrostatic (ion-acoustic) solitary waves in a magnetized plasma with trapped electrons is considered via the Schamel formalism. The direction of propagation is assumed to be arbitrary, i.e., oblique with respect to the magnetic field, for generality. A nonMaxwellian (nonthermal) two-component plasma is considered, consisting of an inertial ion fluid, assumed to be cold for simplicity, and electrons. A (kappa) $\kappa$-type distribution is adopted for the electron population, in addition to particle trapping taken into account in phase space. A damped version of the Schamel-type equation is derived for the electrostatic potential, and its analytical solution, representing a damped solitary wave, is used to examine the nonlinear features of dissipative ion-acoustic solitary waves in the presence of trapped electrons. The influence of relevant plasma configuration parameters, namely the percentage of trapped electrons, the electron superthermality (spectral) index, and the direction of propagation on the solitary wave characteristics is investigated.
\end{abstract}

Keywords: dissipative solitary waves; magnetized plasma; superthermal trapped electrons; kappa distribution; Schamel equation; oblique propagation of electrostatic plasma waves; suprathermals

\section{Introduction}

The occurrence of highly energetic particles is a ubiquitous feature in space plasmas (e.g., in the ionosphere, the auroral zone, solar wind, and at the mesosphere, etc.) and in laboratory plasmas [1-12]. The velocity distribution in such plasmas may deviate from the usual thermal Maxwellian distribution, developing a long-tail for high-velocity arguments due to an excess in the fast (superthermal) part of the population; such a behavior is effectively modeled by a (kappa) $\kappa$-type distribution function [1,4,12-19]. The kappa distribution function was initially postulated by Vasyliunas [1] in an effort to reproduce the observed power-law dependence at high velocities $[17,20,21]$. Since then, a large number of studies adopted kappa distributions, combining theoretical [18,19,22,23], computational [24], and even experimental $[25,26]$ approaches to study the effect of superthermal particle populations on wave dynamics in different plasma environments.

Particle "trapping", i.e., the fact that a portion of, for example, the electron population remains confined in a finite region - thus generating vortices-in phase space, is an intrinsic characteristic of plasma dynamics, often overlooked in studies based on basic fluid theory. Phase-space structures, known as "electron-holes" are thus formed due to particles trapped in the wave potential. This mechanism, initially predicted via kinetic theory [27-29], was later observed in space and in the laboratory [30-36], and it was also shown to occur spontaneously in computer simulations [37]. Of particular relevance to current study is the fact that Simpson et al. [38] reported the presence of trapped electrons in the Saturnian magnetic field, an environment characterized by the existence of $\kappa$-distributed electrons with values of $\kappa \simeq 2-4$, as confirmed by Schippers et al. [34]. It is therefore important to consider the effect of particle nonthermality and trapping effect simultaneously to explore the properties of different electrostatic modes. 
As regards the theoretical modeling of particle trapping, Schamel's original papers [27,28] showed that trapped particles led to a vortex-like electron distribution, and the kinetic model was shown to be associated with a modified version of the known integrable Korteweg-de Vries (KdV) partial differential equation. The "Schamel equation" [28,39-42] describes the evolution of nonlinear electrostatic waves under the influence of a fractional nonlinearity (in contrast with the standard KdV theory where quadratic nonlinearity is dominant). A number of theoretical studies followed [43-49] in an effort to investigate the properties of nonlinear waves (solitary waves, shocks) in the presence of trapped particles, using first principles.

The combined effect of electron superthermality and phase-space trapping was first considered by Williams et al. [40], who adopted the Schamel equation approach to model and characterize ion-acoustic solitary waves in an unmagnetized electron-ion plasma with $\kappa$-distributed electron populations subject to trapping. Following that study, the combined effect of electron superthermality and trapping was considered by Sultana and coworkers [41,42] (on ion-acoustic modes in collisionless plasmas) and by Hassan et al. [50], who investigated the nonlinear features of electron-acoustic waves in a magnetized plasma and considered the combined effect of electron trapping and electron superthermality. The study led by Hassan et al. [50] focused on electron-acoustic waves, a mode known to occur exclusively in the simultaneous presence of two distinct electron populations (usually referred to as the 'cold' and the 'hot' electrons), as it relies in fact on the inertia being provided by the cold electron component and the restoring force being provided by their hot counterpart. The associated (electronic) dynamical frequency scales are clearly distinct from the (slower, ionic) scales that are typical of the study presented here.

To our best knowledge, there is no rigorous and systematic study of the nonlinear propagation of the ion-acoustic waves in a magnetized collisional plasma in the presence of trapped $\kappa$-nonthermal electrons. The investigation at hand is therefore an attempt to fill in this gap by presenting a rigorous and systematic study of the characteristics of ion-acoustic waves propagating in a magnetized $\kappa$-nonthermal plasma [41], taking into account the combined impact of electron trapping and of a suprathermal electron distribution, in account of the inherent plasma collisionality. The main focus here is to investigate the influence of particle trapping on the dynamics of dissipative solitary waves, and also to analyze the effect of the ambient magnetic field and its interplay with wave damping and how these affect the characteristics of obliquely propagating ion-acoustic solitary excitations.

This article is organized as follows. The basic formalism is presented in the following Section 2. A dissipative version of the Schamel equation is derived via a multiscale perturbative approach, and the detail about the nonlinear, dispersion, and dissipative term, is discussed in Section 3. The propagation nature (basic features) of dissipative ion-acoustic waves for different relevant plasma (configuration) parameters is studied numerically in Section 4. Finally, the results obtained are summarized in the concluding Section 5.

\section{Basic Plasma-Fluid-Dynamic Formalism}

An electron-ion plasma is considered here being embedded in a uniform magnetic field directed along the $z$-axis, i.e., $\mathbf{B}_{0}=B_{0} \hat{z}$. Due to their large mass (relative to the electrons), inertial ions are modeled as a cold fluid, i.e., their thermal pressure is neglected for simplicity. At the ionic scale of interest, the electron inertia may be neglected: the electrons are assumed to deviate from thermal equilibrium, and hence, a $\kappa$-type distribution will be explicitly adopted to model their distribution. For the purpose of this analysis, the combined effect of electron trapping and superthermality is considered, following the steps outlined in Ref. [40].

Charge neutrality at equilibrium imposes: $z_{i} n_{i 0}-n_{e 0}=0$, where $n_{i 0}$ and $n_{e 0}$ denote the unperturbed ion and electron number densities, respectively, while $z_{i}$ is the charge state of the ion component (e.g., 1, 2,...; the value of $z_{i}$ is left arbitrary here, for generality). 
We are interested in modeling the dynamics of ion-acoustic excitations, whose phase speed $v_{p h}$ may well exceed the ion thermal speed, but is far smaller than the electron thermal speed. The following fluid evolution equations are considered:

$$
\begin{aligned}
\frac{\partial n_{i}}{\partial T}+\nabla \cdot\left(n_{i} \mathbf{u}_{i}\right) & =0 \\
\frac{\partial \mathbf{u}_{i}}{\partial T}+\left(\mathbf{u}_{i} \cdot \nabla\right) \mathbf{u}_{i} & =-\frac{z_{i} e}{m_{i}} \nabla \Phi \\
& +\frac{z_{i} e B_{0}}{m_{i}}\left(\mathbf{u}_{i} \times \hat{z}\right)-v_{i} \mathbf{u}_{i} \\
\nabla^{2} \Phi & =4 \pi e\left(n_{e}-z_{i} n_{i}\right)
\end{aligned}
$$

where $n_{i}\left(n_{e}\right)$ denotes the number density of the ion (electron) species, $\mathbf{u}_{i}$ is the ion fluid speed, $\Phi$ is the electrostatic wave potential (all these quantities are dynamic functions of space and time), and $e$ is electron charge. An ad hoc damping term is introduced in the fluid equation of motion (momentum conservation equation) to account for, e.g., ion-neutral collisions; the collision frequency $v_{i}$ was defined to this effect.

The combined effect of electron trapping and deviation from Maxwell-type equilibrium was studied analytically in Ref. [40]; the tedious algebraic procedure need not be reproduced here, but the main steps are summarized. A modified $\kappa$-distribution function, effectively taking into account the trapped part of the electron population (i.e., for electrons trapped in the wave potential if their energy $E_{e}<0$ ) is given by [40]

$$
\begin{aligned}
f(v, \phi) & =\frac{\Gamma(\kappa)}{\sqrt{2 \pi}(\kappa-3 / 2)^{1 / 2} \Gamma(\kappa-1 / 2)} \\
& \times\left[1+\beta\left(\frac{v^{2} / 2-\phi}{\kappa-3 / 2}\right)\right]^{-\kappa} \text { for } E_{e} \leq 0 .
\end{aligned}
$$

Here, $v$ is the velocity, $\phi$ is the electrostatic potential, and $\kappa$ is the superthermality index (measures deviation from the Maxwell-Boltzmann distribution), while $\beta(<1)$ quantifies the efficiency of electron trapping. The known vortex-type distribution for trapped Maxwellian electrons is recovered in the limit $\kappa \rightarrow \infty$. The number density of the electrons is obtained by integration as [40]

$$
\begin{aligned}
n_{e}(\phi) & =\int_{-\infty}^{-\sqrt{2 \phi}} f_{e}^{\kappa}(v, \phi) d v+\int_{-\sqrt{2 \phi}}^{\sqrt{2 \phi}} f(v, \phi) d v \\
& +\int_{\sqrt{2 \phi}}^{\infty} f_{e}^{\kappa}(v, \phi) d v,
\end{aligned}
$$

where $f_{e}^{\kappa}(v, \phi)$ is the $\kappa$-distribution function for the free electrons; details can be found in Ref. [17].

By normalizing all variables, one obtains the following system of (dimensionless) equations:

$$
\begin{aligned}
& \frac{\partial n}{\partial t}+\tilde{\nabla} \cdot(n \mathbf{u})=0, \\
& \frac{\partial \mathbf{u}}{\partial t}+(\mathbf{u} . \tilde{\nabla}) \mathbf{u}=-\tilde{\nabla} \phi+\Omega_{c}(\mathbf{u} \times \hat{z})-v \mathbf{u}, \\
& \tilde{\nabla}^{2} \phi \simeq 1-n+a_{1} \phi+a_{2} \phi^{3 / 2}+a_{3} \phi^{2},
\end{aligned}
$$

where $n=n_{i} / n_{i 0}, u=\left[m_{i} u_{i}^{2} /\left(z_{i} T_{e}\right)\right]^{1 / 2}$ with $m_{i}$ being the mass of ion, $T_{e}$ being the electron temperature (Boltzmann's constant $k_{B}$ is omitted where obvious), the electrostatic potential $\phi=e \Phi / T_{e}, \lambda_{D}=\left(T_{e} / 4 \pi e^{2} z_{i} n_{i 0}\right)^{1 / 2}, t=\omega_{p, i} T$ (where $\omega_{p, i}=\left(4 \pi e^{2} z_{i}^{2} n_{i 0} / m_{i}\right)^{1 / 2}$ is the ion plasma frequency, and $T$ is the inverse of the ion plasma frequency), $\Omega_{c}=\omega_{c, i} / \omega_{p, i}$ 
(where $\omega_{c, i}=z_{i} e B_{0} / m_{i}$ ) is the reduced cyclotron frequency, and $v=v_{i} / \omega_{p, i}$ (note that all frequencies were scaled by the ion plasma frequency for convenience). The information related to electron trapping, for $\kappa$-distributed electrons, is "hidden" in the coefficients $a_{1}, a_{2}$, $a_{3}$ entering the normalized expression of Poisson Equation (8), which are given by [40]

$$
\begin{aligned}
& a_{1}=\frac{2 \kappa-1}{2 \kappa-3}, \quad a_{2}=\frac{8 \sqrt{2 / \pi}(\beta-1) \kappa \Gamma(\kappa)}{3(2 \kappa-3)^{3 / 2} \Gamma(\kappa-1 / 2)}, \\
& a_{3}=\frac{4 \kappa^{2}-1}{2(2 \kappa-3)^{2}} .
\end{aligned}
$$

Once a solution for the electrostatic potential $\phi$ is formally obtained, the trapped electron population's density obtained from Equation (5) can be expressed as [40]

$$
n_{e} \simeq 1+a_{1} \phi+a_{2} \phi^{3 / 2}+a_{3} \phi^{2} \cdots
$$

which to be substituted into Poisson Equation (8). The known analogous expression for the trapped electron number density in the case of Maxwellian plasma [28] is readily recovered here, upon considering the limit $\kappa \rightarrow \infty$ in the latter relation. On the other hand, the limit of Equation (10) as $\beta \rightarrow 1$ leads to the classical expression for $\kappa$-distributed electrons; see e.g., Ref. [51] and elsewhere. Finally, the Maxwellian limit for free electrons, viz. $a_{1}=2 a_{3}=1$, is recovered by considering $\beta \rightarrow 1$ and $\kappa \rightarrow \infty$, reducing the electron density dependence to $\mathrm{e}^{\phi} \simeq 1+\phi+\phi^{2} / 2$, as expected.

The closed system of Equations (6)-(8), describes the evolution of the plasma (fluid) state variables, and forms the basis of the analysis here.

\section{A Schamel Equation for Damped Ion-Acoustic Waves (IAWs)}

To model small-amplitude ion-acoustic excitations (dissipative solitary waves) within the model under consideration, one needs to proceed by defining a set of stretched coordinates as

$$
\xi=\epsilon^{1 / 4}\left(l_{x} \hat{x}+l_{y} \hat{y}+l_{z} \hat{z}-v_{p} t\right), \quad \tau=\epsilon^{3 / 4} t,
$$

where $\epsilon(\ll 1)$ is a small parameter that measures the strength of the nonlinearity, $v_{p}$ is a constant to be determined (in fact, representing the phase speed, scaled by the ion sound speed, $\left.c_{0}=\left(z_{i} T_{e} / m_{i}\right)^{1 / 2}\right)$, and $l_{x}, l_{y}$ and $l_{z}$, are directional cosines of the wave vector $\mathbf{k}$ along the $x, y$ and $z$ axes, respectively (for instance, $l_{z}=(\mathbf{k} \cdot \hat{z}) / k$ ), hence $l_{x}^{2}+l_{y}^{2}+l_{z}^{2}=1$. Let us recall that the position variables $x, y$ and $z$ are all normalized by $\lambda_{D}$, while $\tau$ is normalized by the ion plasma period $\omega_{p, i}^{-1}$. The above Ansatz, which was first introduced in Ref. [28] and then later adopted by various authors (e.g., [40,52]), essentially describes a Galilean transformation into a slowly varying moving frame, wherein the time variation of the structure is even slower in time.

The dependent variables $n, \mathbf{u}$ and $\phi$ may now be expanded near the equilibrium states as power series of $\epsilon$ as follows:

$$
\left.\begin{array}{l}
n=1+\epsilon n_{1}+\epsilon^{3 / 2} n_{2}+\ldots, \\
u_{x}=\epsilon^{5 / 4} u_{1, x}+\epsilon^{3 / 2} u_{2, x}+\ldots, \\
u_{y}=\epsilon^{5 / 4} u_{1, y}+\epsilon^{3 / 2} u_{2, y}+\ldots, \\
u_{z}=\epsilon u_{1, z}+\epsilon^{3 / 2} u_{2, z}+\ldots, \\
\phi=\epsilon \phi_{1}+\epsilon^{3 / 2} \phi_{2}+\ldots
\end{array}\right\}
$$

To close the series expansion of the variables, a weak dissipation [53-55] to be considered due to ion-neutral collisions by assuming that the damping coefficient scales as $v=\epsilon^{3 / 4} v_{0}$.

Let us now proceed by substituting expansions (11) and (12) into the considered fluid plasma model Equations (6)-(8) and collecting various terms arising in each order in $\epsilon$. 
The phase speed $v_{p}$ is obtained as a compatibility constraint upon considering the lowest order contributions in $\epsilon$ from each of the equations; the resulting expression reads:

$$
v_{p}=l_{z} / \sqrt{a_{1}} .
$$

This expression for the phase speed $v_{p}$ depends on the angle $\theta$ (via $l_{z}=\cos \theta$ ) and on the electron superthermality index $\kappa$. Considering parallel propagation $\left(l_{z}=1\right)$, the known expression for the ion sound speed in nonmagnetized plasma [22,40] is recovered as expected. Recovering dimensions for a minute, for physical transparency, Equation (13) leads to

$$
V_{p}=\frac{\omega}{k}=\lambda_{D} \omega_{p, i} \frac{l_{z}}{\sqrt{a_{1}}}=\left(\frac{z_{i} T_{e}}{m_{i}}\right)^{1 / 2}\left(\frac{2 \kappa-3}{2 \kappa-1}\right)^{1 / 2} l_{z},
$$

where $\omega, k$ and $V_{p}$ here denote the wave (angular) frequency, the wavenumber and the phase speed (in the dimensional form), respectively. The acoustic ("sound") speed is thus recovered for infinitely large $\kappa$, while a lower value (i.e., predicting slower solitary waves) is predicted for small $\kappa$, in agreement with earlier theoretical predictions [22] and with space observations [21].

The variation of the ion-acoustic phase speed, $v_{p}$, versus the electron's superthermality index, $\kappa$, is depicted in Figure 1, suggesting a slower phase speed in a plasma with significant portion of the electrons in the superthermal region (i.e., lower values of $\kappa$ ), when compared with the case of thermal (Maxwellian) electron. The phase speed is higher for parallel propagation than for oblique propagation, as shown in Figure 1. As expected, the curve tends to unity, asymptotically $\left(v_{p} \rightarrow 1\right)$ for infinite kappa and for parallel propagation, prescribing the acoustic speed (in electron-ion plasma) as the phase speed in the Maxwellian limit.

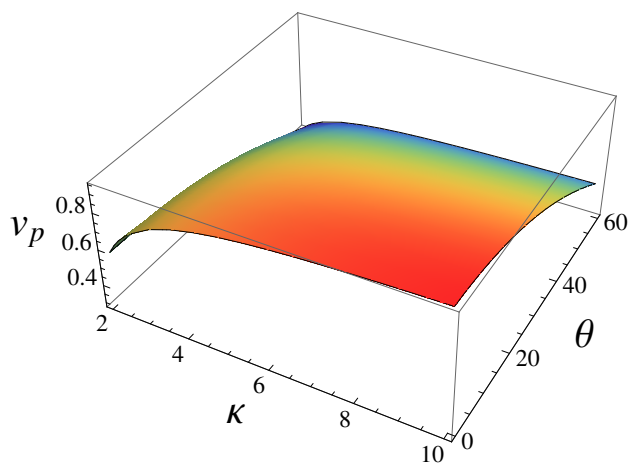

Figure 1. Phase speed, $v_{p}$, versus electron superthermality index, $\kappa$, and obliqueness angle, $\theta=\cos ^{-1} l_{z}$, where $l_{z}$ is a directional cosine of the wave vector $\mathbf{k}$ along $z$-axis.

The perpendicular ( $x$ and $y$ ) components of the electric field related drift of the ion fluid, in terms of the electric potential $\phi_{1}$, can be obtained by separating the $y$ and $x$ components of the momentum equation, respectively, as

$$
\begin{aligned}
u_{1, x} & =-\frac{l_{y}}{\Omega_{c}} \frac{\partial \phi_{1}}{\partial \xi}, \\
\text { and } \quad & u_{1, y}=\frac{l_{x}}{\Omega_{c}} \frac{\partial \phi_{1}}{\partial \xi} .
\end{aligned}
$$

Following an analogous procedure, the parallel $(z)$ component of the ion fluid velocity is obtained as

$$
u_{1, z}=\frac{l_{z}}{v_{p}} \phi_{1},
$$


Finally, in leading order, the perturbation of the ion density $n \simeq 1+\epsilon n_{1}+\mathcal{O}\left(\epsilon^{3 / 2}\right)$ is obtained as

$$
n_{1}=\left(\frac{l_{z}}{v_{p}}\right)^{2} \phi_{1}
$$

The next order in $\epsilon$ (obtained upon separating $\epsilon^{3 / 2}$ from the momentum equation) leads to the $x$ and $y$-components of the second order drift velocity of the ion fluid in the form

$$
\begin{aligned}
& u_{2, x}=\frac{l_{x} v_{p}}{\Omega_{c}^{2}} \frac{\partial^{2} \phi_{1}}{\partial \xi^{2}} \\
& u_{2, y}=\frac{l_{y} v_{p}}{\Omega_{c}^{2}} \frac{\partial^{2} \phi_{1}}{\partial \xi^{2}}
\end{aligned}
$$

Following the same procedure (i.e., separating coefficient of $\epsilon^{7 / 4}$ from the continuity and the $z$-component of the momentum equations, and then $\epsilon^{3 / 2}$ from the Poisson equation) and eventually eliminating $n_{2}, u_{2, z}$, and $\phi_{2}$, one is led to a nonlinear partial differential equation (PDE) in the form

$$
\frac{\partial \psi}{\partial \tau}+A \psi^{1 / 2} \frac{\partial \psi}{\partial \xi}+B \frac{\partial^{3} \psi}{\partial \xi^{3}}+C \psi=0,
$$

where, for brevity, the leading contribution of the electrostatic potential is denoted by $\psi=\phi_{1}$.

Equation (21), which bears the structure of the original Schamel equation [28], with the addition of the last term (arising due to collisions being taken into account), represents an evolution equation for an electrostatic potential disturbance, $\phi \simeq \epsilon \psi+\mathcal{O}\left(\epsilon^{3} / 2\right)$, in a region where the trapped electrons are present. The algebraic scheme implied is obvious: once $\psi$ is obtained from Equation (21), the leading contributions for the ion density and for the ion fluid speed (three) components can be obtained from (four) Equations (15)-(18).

The nonlinearity coefficient $A$, which is responsible for wave steepening, is given by

$$
A=-\frac{3}{4} \frac{v_{p}^{3}}{l_{z}^{2}} a_{2}=-\frac{3}{4} \frac{a_{2}}{a_{1}^{3 / 2}} l_{z}
$$

The nonlinearity dependence enters via both $\theta$ and $\kappa$, as expected: this is seen in Figure 2a.

On the other hand, the dispersion coefficient $B$-which is responsible for wave broadening-is given by

$$
B=\frac{v_{p}^{3}}{2 l_{z}^{2}}\left(1+\frac{1-l_{z}^{2}}{\Omega_{c}^{2}}\right) .
$$

The expression for the coefficient $B$ can be simplified upon setting $v_{p}^{3} / 2 l_{z}^{2}=l_{z} / 2 a_{1}^{3 / 2}$, showcasing the dependence of $B$ on the propagation angle (via $l_{z}$ ) and on $\kappa$ (via $a_{1}$ ), as shown in Figure $2 b$. The influence of the magnetic field (via $\Omega_{\mathcal{C}}$ ) disappears in the case of parallel propagation $\left(l_{z}=1\right)$, thus recovering a one-dimensional damped Schamel equation for unmagnetized plasma (this was intuitively expected, since the Larmor force has no component in the direction of the magnetic field, and thus does not affect parallel wave propagation).

Finally, the dissipative term $C$ is given by

$$
C=\frac{v_{0}}{2}
$$

as imposed by compatibility requirements (i.e., balancing various terms occurring in the same order in $\epsilon$ ). 
(a)

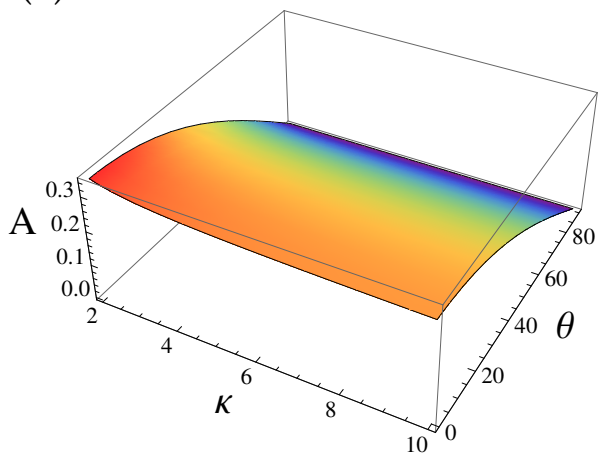

(b)

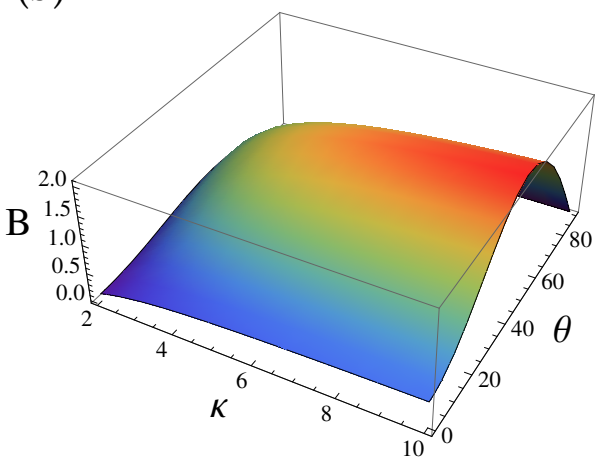

Figure 2. Nonlinearity term $A(\mathbf{a})$ and dispersion term $B(\mathbf{b})$ versus $\kappa$ and obliqueness angle $\theta$ for $\beta=0.5$ and $\Omega_{c}=0.3$, where $\beta$ denotes the efficiency of electron trapping and $\Omega_{c}$ is the ratio of the the reduced cyclotron frequency to the ion plasma frequency.

Interestingly, both $A$ and $B$ vanish for perpendicular propagation (i.e., for $l_{z}=\cos (\pi / 2)=0$ ), as seen in Figure 2. On the other hand, considering parallel propagation $\left(l_{z}=\cos (0)=1\right.$ ) and the Maxwellian limit (infinite $\kappa$ ), one finds $v_{p}=1$ (acoustic speed), while the two coefficients become $A=(1-\beta) / \sqrt{\pi}$ and $B=1 / 2$, thus recovering exactly the analytical form of the original Schamel equation [28]. Figure 2 examines the influence of superthermality index $\kappa$ and the obliqueness $\theta$ on the nonlinear term $A$ and the dispersion term $B$. One can see that the (value of the) nonlinearity term increases, while the dispersive term decreases, if one assumes stronger deviation from the Maxwellian equilibrium, i.e., for small value of the $\kappa$ parameter. On the other hand, for fixed $\kappa$, the nonlinearity term $A$ attains its highest value for parallel propagation $(\theta=0)$, as shown in Figure 2.

The dispersive term shows slightly more perplex behavior by increasing with growing $\theta$, reaching a maximum, and then going to zero-as said above-for $\theta=\pi / 2$. It is evident in Equation (22) and in Figure 3a that $a_{2} \rightarrow 0$, and, hence, $A \rightarrow 0$ in the limit $\beta \rightarrow 1$; therefore, the nonlinear Equation (21) is not valid in the absence of trapped electrons. On the other hand, the dispersive term decreases with stronger magnetic field, as seen in Figure $3 b$.

(a)

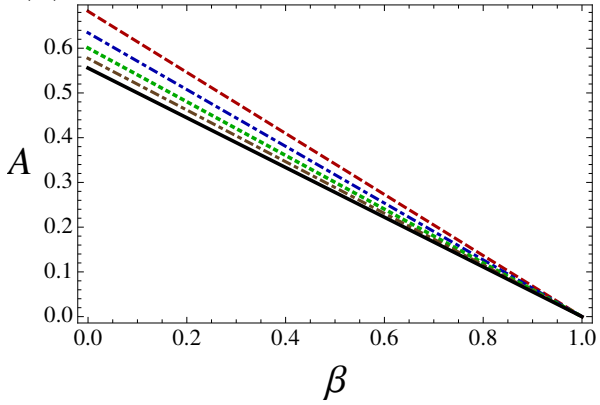

(b)

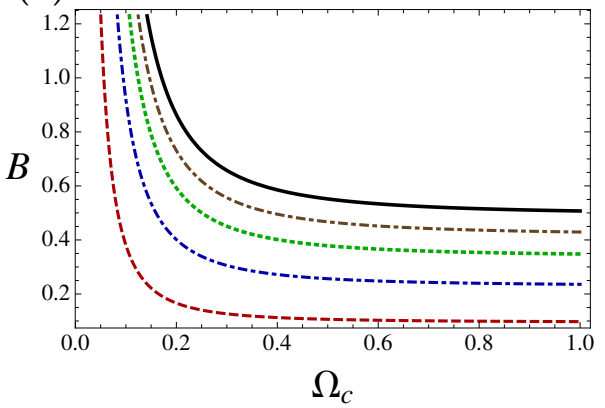

Figure 3. (a) Nonlinearity term $A$ versus $\beta$. A does not depend on the magnetic field. (b) Dispersion term $B$ versus $\Omega_{c}$. $B$ does not depend on $\beta . \theta=10^{\circ}$ is assumed and $\kappa=2,3,5,10$, and $\infty$ (top to bottom in (a) and bottom to top in (b)).

\section{Parametric Analysis}

In this Section, we are interested in tracing the influence of different plasma configuration parameters, such as the superthermality (spectral) index, $\kappa$, the electron trapping parameter, $\beta$, the collisional term, $\nu$, the obliqueness angle, $\theta$, and the ambient magnetic field (strength), $B_{0}$, on the propagation characteristics of ion-acoustic solitary waves within the model under consideration. To see how these plasma configuration parameters affect the dynamical properties of solitary waves, first, dissipative effect is assumed to be negligi- 
ble: $v \rightarrow 0$. The damped Schamel Equation (21) then reduces to a $\kappa$-dependent form of the Schamel-type equation [28], which possesses a solitary wave solution in the form [28,40]

$$
\psi(\xi, \tau)=\psi_{0} \operatorname{sech}^{4}\left(\frac{\xi-u_{0} \tau}{\delta}\right)
$$

representing a pulse-shaped excitation with amplitude $\psi_{0}=\left(15 u_{0} / 8 A\right)^{2}$, width $\delta=$ $\sqrt{16 \mathrm{~B} / u_{0}}$ and velocity $u_{0}$ in the moving reference frame (note that the actual speed in the laboratory frame is $v_{p}+\epsilon u_{0}$, so the pulse structure is superacoustic; recall that $v_{p}$ is essentially the sound speed). The product $\psi_{0} \delta^{4}=(30 \mathrm{~B} / A)^{2}$ is constant for a given (fixed) set of plasma parameters, that is in fact independent of $u_{0}$. The electric field $E(=-\nabla \psi)$ which is associated with the solitary potential in Equation (25) is of the form:

$$
E=-E_{0} \operatorname{sech}^{4}\left(\frac{\xi-u_{0} \tau}{\delta}\right) \tanh \left(\frac{\xi-u_{0} \tau}{\delta}\right)
$$

where $E_{0}=225 u_{0}^{2} /\left(16 A^{2} \delta\right)$ (this is actually the norm of the vector; the respective components are regulated by the direction cosines $l_{x, y, z} ;$ recall that $l_{x}^{2}+l_{y}^{2}+l_{z}^{2}=1$ ). The pulse form for the potential is shown in Figure 4, while the associated bipolar electric field structures are shown in Figure 5 b,d.

To trace the dynamical evolution of the solitary wave solution and to elucidate the role of different plasma configuration parameters on the properties of (nonlinear) solitary waves, the nonlinear damped Schamel Equation (21) was solved numerically by using the Wolfram MATHEMATICA ${ }^{\mathrm{TM}}$ software package, adopting the solitary wave solution (25) as initial condition.

(a)

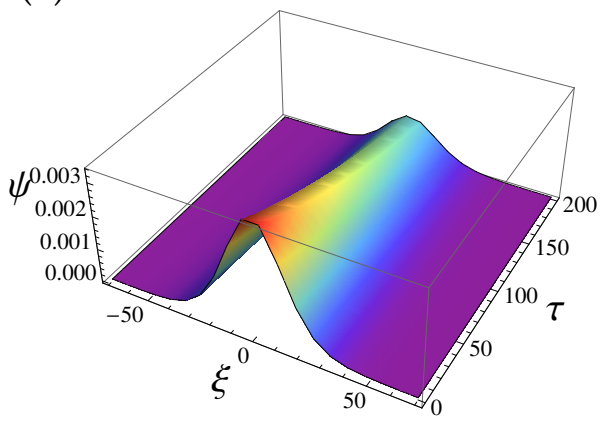

(b)

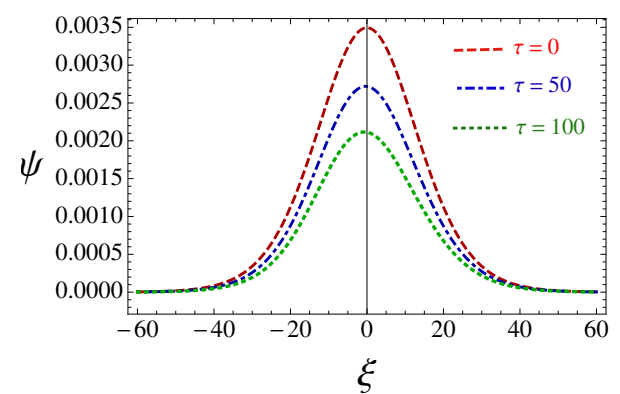

Figure 4. (a) The ion-acoustic solitary potential pulse $\psi$ versus the space coordinate $\xi$ and the time $\tau$. (b) Potential pulse versus $\xi$ at different time instants. Here, $\beta=0.5, \kappa=3, \Omega_{c}=0.2, v=0.01$, $\theta=10^{\circ}$, and $u_{0}=0.01$ are used. See text for details.

The time evolution of dissipative ion-acoustic solitary potential waveforms (pulses) is shown in Figure 4. The analytical solution (25) was adopted at an initial condition, and then Equation (21) was solved numerically for $v \neq 0$. As expected, the pulse amplitude decreases in time due to the damping, as illustrated in Figure 4.

The influence of the trapping parameter $\beta$ and also of the superthermality (spectral) index $\mathcal{K}$ was investigated numerically; a snapshot at $\tau=50$ (dimensionless units) is shown in Figure 5. Here, $\Omega_{c}=0.2, v=0.01, u_{0}=0.01, \tau=50$, and $\theta=10^{\circ}$ are used. One can see that both the height and the width of the solitary wave are affected by the trapping parameter $\beta$ (Figure 5a) and by the value of $\kappa$ (Figure $5 c$ ). As $\beta$ increases, the waves become taller in amplitude, but the width remains unchanged; see Figure 5a. An increase in plasma superthermality (that is, a smaller value of $\kappa$ ) results in shorter and narrower solitary waves, as seen in Figure 5c. These results recover the theoretical predictions of Ref. [41] for the collision-free case, i.e., for $v=0$.

A similar investigation is shown in Figure 6, where the solitary wave solution (25) was obtained numerically for $\kappa=3, \beta=0.5, u_{0}=0.01, \tau=50$, and $\theta=10^{\circ}$. The role of the 
magnetic field (strength) $B_{0}$ (via $\Omega_{\mathcal{c}}$ ) is shown in Figure 6a. As $B_{0}$ increases, the width of the solitary wave decreases, while the amplitude is unaffected.

Finally, in Figure $6 b$, various values of the collisional parameter $v$ are considered (keeping all other values fixed). As expected, the pulse amplitude decreases with higher dissipation.

(a)

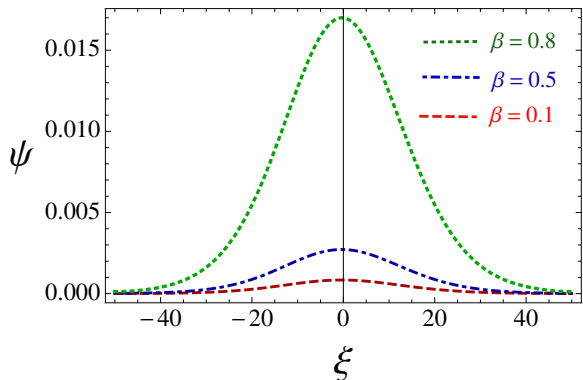

(c)

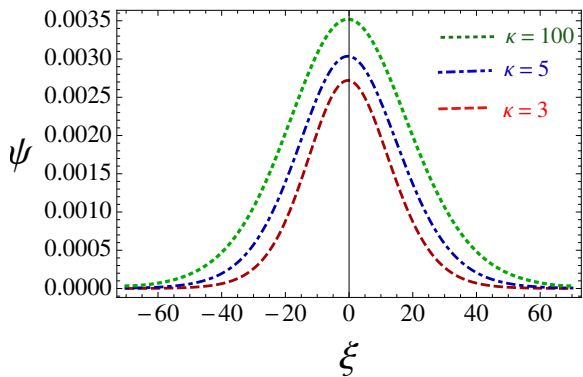

(b)

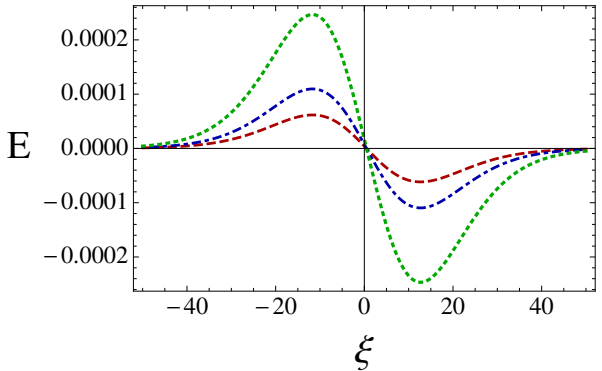

(d)

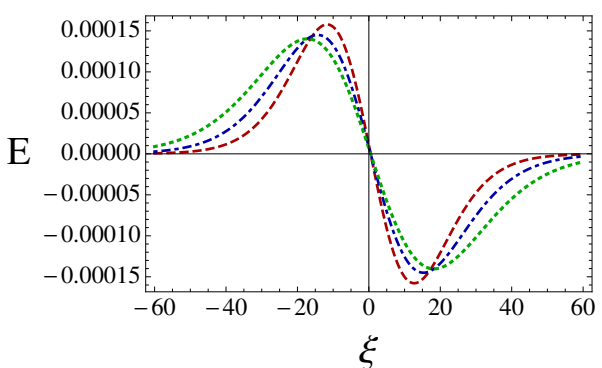

Figure 5. (a) Effect of trapping parameter $\beta$ on electrostatic solitary wave (pulse) profile and (b) associated electric field structures for $\kappa=3$. (c) Effect of superthermality index $\kappa$ on solitary pulse and (d) associated electric field for $\beta=0.5$. Here, $\Omega_{\mathcal{c}}=0.2, v=0.01, u_{0}=0.01, \tau=50$, and $\theta=10^{\circ}$ are used.

(a)

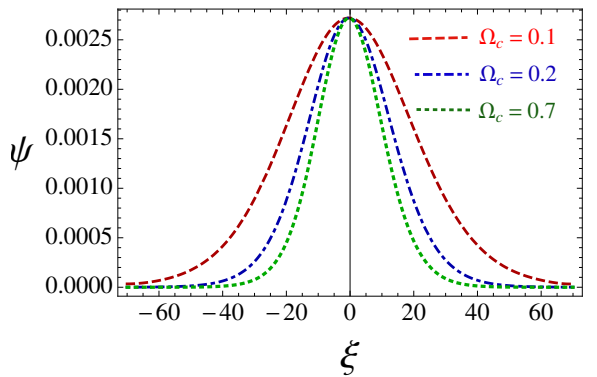

(b)

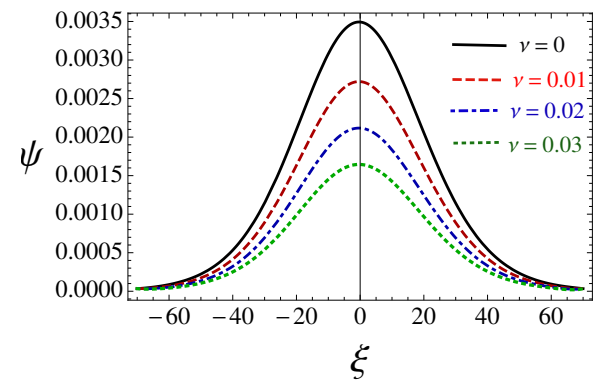

Figure 6. Effect of (a) external magnetic field $\Omega_{c}$ (for $v=0.01$ ) and of (b) collisionality parameter $v$ (for $\Omega_{c}=0.1$ ) on electrostatic solitary waves (pulses) for $\kappa=3, \beta=0.5, u_{0}=0.01, \tau=50$, and $\theta=10^{\circ}$.

\section{Conclusions}

In this paper, the basic features of damped ion-acoustic solitary waves were investigated in the presence of trapped superthermal electrons described by a $\kappa$-type (nonMaxwellian) distribution [40]. The effect of ion-neutral collisions was also taken into account, leading to wave damping as expected.

The reductive perturbation approach were adopted to derive a nonlinear Schameltype partial differential equation featuring an additional damping term. The solitary wave solution of the standard (nondissipative) Schamel equation was used to solve the 
damped Schamel equation numerically and to analyze the basic features of dissipative ion-acoustic solitary waves. The amplitude of solitary waves was found to decrease, while their width becomes narrower with an increase in superthermality (i.e., for a stronger deviation from Maxwellian equilibrium). The proportion of trapped electrons also affects solitary waves, since their amplitude increases in the presence of a larger proportion of trapped electrons in the plasma; on the other hand, rather counter-intuitively, their width remains the same. The above behavior was also observed via numerical integration of the dissipative Schamel equation.

While the nonlinear term is independent of the external magnetic field, the dispersive term depends strongly on the external magnetic field and in fact decreases for strong magnetic field (strength) values. Therefore, a steeper solitary wave with same maximum amplitude will be expected to occur in the presence of a stronger magnetic field, as confirmed by the numerical simulation here.

The current study focused on the 'simplest' version of a fluid model for magnetized plasma, i.e., assuming a uniform magnetic field and neglecting drift forces. A drift-kinetic approach would require the electrons to create a more complete picture to correctly account for the non-negligible $E \times B$ drift, for example. These aspects, as investigated, e.g., by Jovanoviç et al. [56], and summarized by Eliasson and Shukla [57], lie beyond the scope of the present study (weak $\sim \epsilon$ excitations were considered here which, in addition to the absence of an ambient electric field (bias), prescribe a negligible $E \times B$ drift).

The fundamental trapping scenario was considered in this paper, (the so-called $\beta$ trapping effect). It may occur, however, that the filamentation process in the final state of pattern formation in the electron phase space results in multiple electron transfer taking place through the separatrix; in turn, leading to additional trapping scenarios. In that case, the electric wave potential may not be expressed in a closed algebraic form, and new types of nonlinear structures may arise, as recently pointed out by Schamel [58,59]. As argued there, the existing wave theory for phase space holes, based on the linear Landau-van Kampen approach, overlooks these trapping and coherence aspects in pattern formation, and thus fails to account for a plethora of nonlinear phenomena, which are nonetheless predicted by this new approach $[58,59]$. Covering these aspects may form the focus of future studies.

The results, obtained here, aim to contribute to the understanding of the salient features of nonlinear electrostatic perturbations in non-Maxwellian plasmas, in account of electron trapping in phase space.

Author Contributions: Conceptualization, S.S.; formal analysis, S.S.; supervision, I.K.; writingoriginal draft, S.S. All authors have read and agreed to the published version of the manuscript.

Funding: Financial support by the project FSU-2021-012/8474000352 "Modeling of Nonlinear Waves and Shocks in Space and Laboratory Plasmas" (PI: Ioannis Kourakis), funded by Khalifa University of Science and Technology (Abu Dhabi, United Arab Emirates), is acknowledged, with thanks. Support from ADEK (Abu Dhabi Department of Education and Knowledge, United Arab Emirates), currently ASPIRE, in the form of an AARE-2018 (ADEK Award for Research Excellence 2018) grant (ADEK/HE/157/18-AARE-179) is also gratefully acknowledged.

Data Availability Statement: Not applicable.

Acknowledgments: Both authors had the pleasure and honor to have collaborated with Reinhard Schlickeiser on various projects in the past. This work is therefore dedicated to Reinhard Schlickeiser on the occasion of his 70th birthday. As a renowned plasma physicist and as an efficient supervisor, collaborator, and colleague, Reinhard Schlickeiser was a source of inspiration for both of us.

Conflicts of Interest: The authors declare no conflict of interest. 


\section{References}

1. Vasyliunas, V.M. A survey of low-energy electrons in the evening sector of the magnetosphere with OGO 1 and OGO 3. J. Geophys. Res. 1968, 73, 2839-2884. [CrossRef]

2. De Feiter, L.D.; De Jager, C. Superthermal plasma nodules and their relation to solar flares. Sol. Phys. 1973, 28, 183-186. [CrossRef]

3. Scudder, J.D.; Sittler, E.C.; Bridge, H.S. A survey of the plasma electron environment of Jupiter: A view from Voyager. J. Geophys. Res. 1981, 86, 8157-8179. [CrossRef]

4. Leubner, M.P. On Jupiter's whistler emission. J. Geophys. Res. 1982, 87, 6335-6338. [CrossRef]

5. Christon, S.P.; Williams, D.J.; Mitchell, D.G.; Frank, L.A.; Huang, C.Y. Spectral characteristics of plasma sheet ion and electron populations during undisturbed geomagnetic conditions. J. Geophys. Res. 1989, 94, 13409-13424. [CrossRef]

6. Collier, M.R. On generating kappa-like distribution functions using velocity space Lévy flights. Geophys. Res. Lett. 1993, 20, 1531-1534. [CrossRef]

7. Decker, D.T.; Basu, B.; Jasperse, J.R.; Strikland, D.J.; Sharber, J.R.; Winningham, J.D. Upgoing electrons produced in an electronproton-hydrogen atom aurora. J. Geophys. Res. 1995, 100, 21409-21420. [CrossRef]

8. Codrescu, M.V.; Fuller-Rowell, T.J.; Robble, R.G.; Evans, D.S. Medium energy particle precipitation influences on the mesosphere and lower thermosphere. J. Geophys. Res. 1997, 102, 19977-19987. [CrossRef]

9. Maksimovic, M.; Gary, S.P.; Skoug, R.M. Solar wind electron suprathermal strength and temperature gradients: Ulysses observations. J. Geophys. Res. 2000, 105, 18337-18350. [CrossRef]

10. Antonova, E.E.; Stepanova, M.V.; Teltzov, M.V.; Tverskoy, B.A. Multiple inverted-V structures and hot plasma pressure gradient mechanism of plasma stratification. J. Geophys. Res. 1998, 103, 9317-9332. [CrossRef]

11. Mori, H.; Ishii, M.; Murayama, Y.; Kubota, M.; Sakanoi, K.; Yamamoto, M.Y.; Monzen, Y.; Lummerzheim, D.; Watkins, B.J. Energy distribution of precipitating electrons estimated from optical and cosmic noise absorption measurements. Ann. Geophys. 2004, 22, 1613-1622. [CrossRef]

12. Livadiotis, G. (Ed.) Kappa Distributions. Theory and Applications in Plasmas; Elsevier: Amsterdam, The Netherlands, 2017.

13. Armstrong, T.P.; Paonessa, M.T.; Bell, E.V.; Krimigis, S.M. Voyager observations of Saturnian ion and electron phase space densities. J. Geophys. Res. 1983, 88, 8893-8904. [CrossRef]

14. Hasegawa, A.; Mima, K.; Duong-van, M. Plasma Distribution function in a superthermal radiation field. Phys. Rev. Lett. 1985, 54, 2608-2611. [CrossRef]

15. Hellberg, M.A.; Mace, R.L.; Armstrong, R.J.; Karlstad, G. Electron-acoustic waves in the laboratory: An experiment revisited. J. Plasma Phys. 2000, 64, 433-443. [CrossRef]

16. Baluku, T.K.; Hellberg, M.A. Dust acoustic solitons in plasmas with kappa-distributed electrons and/or ions. Phys. Plasmas 2008, 15, 123705. [CrossRef]

17. Hellberg, M.A.; Mace, R.L.; Baluku, T.K.; Kourakis, I.; Saini, N.S. Comment on “Mathematical and physical aspects of Kappa velocity distribution" [Phys. Plasmas 14, 110702 (2007)]. Phys. Plasmas 2009, 16, 094701. [CrossRef]

18. Sultana, S.; Kourakis, I.; Saini, N.S.; Hellberg, M.A. Oblique electrostatic excitations in a magnetized plasma in the presence of excess superthermal electrons. Phys. Plasmas 2010, 17, 032310. [CrossRef]

19. Sultana, S.; Kourakis, I. Electrostatic solitary waves in the presence of excess super-thermal electrons: Modulational instability and envelope soliton modes. Plasma Phys. Control. Fusion 2011, 53, 045003. [CrossRef]

20. Mauk, B.H.; Mitchell, D.G.; McEntire, R.W.; Paranicas, C.P.; Roelof, E.C.; Williams, D.J.; Krimigis, S.M.; Lagg, A. Energetic ion characteristics and neutral gas interactions in Jupiter's mag-netosphere. J. Geophys. Res. 2004, 109, A09S12.

21. Hapgood, M.; Perry, C.; Davies, J.; Denton, M. The role of suprathermal particle meas-urements in CrossScale studies of collisionless plasma processes. Planet. Space Sci. 2011, 59, 618-629. [CrossRef]

22. Kourakis, I.; Sultana, S.; Hellberg, M.A. Dynamical characteristics of solitary waves, shocks and envelope modes in kappadistributed non-thermal plasmas: An overview. Plasma Phys. Control. Fusion 2012, 54, 124001. [CrossRef]

23. Atteya, A.; Sultana, S.; Schlickeiser, R. Dust-ion-acoustic solitary waves in magnetized plasmas with positive and negative ions: The role of electrons superthermality. Chinese J. Phys. 2018, 56, 1931-1939. [CrossRef]

24. Lotekar, A.; Kakad, A.; Kakad, B. Generation of ion acoustic solitary waves through wave breaking in superthermal plasmas. Phys. Plasma 2017, 24, 102127. [CrossRef]

25. Goldman, M.V.; Newman, D.L.; Mangeney, A. Theory of weak bipolar fields and electron holes with applications to space plasmas. Phys. Rev. Lett. 2007, 99, 145002. [CrossRef]

26. Sarri, G. Observation and characterization of laser-driven phase space electron holes. Phys. Plasmas 2010, 17, 010701. [CrossRef]

27. Schamel, H. Stationary solitary, snoidal and sinusoidal ion acoustic waves. Plasma Phys. 1972, 14, 905-924. [CrossRef]

28. Schamel, H. A modified Korteweg-de Vries equation for ion acoustic wavess due to resonant electrons. J. Plasma Phys. 1973, 9, 377-387. [CrossRef]

29. Schamel, H. Cnoidal electron hole propagation: Trapping, the forgotten nonlinearity in plasma and fluid dynamics. Phys. Plasma 2012, 19, 020501. [CrossRef]

30. Ergun, R.E.; Carlson, C.W.; McFadden, J.P.; Mozer, E.S.; Delory, G.T.; Peria, W.; Chaston, C.C.; Temerin, M.; Roth, I.; Muschietti, L.; et al. FAST satellite observations of large-amplitude solitary structures. Geophys. Res. Lett. 1998, 25, 2041-2044. [CrossRef]

31. Ergun, R.E.; Andersson, L.; Main, D.S.; Su, Y.J.; Carlson, C.W.; McFadden, J.P.; Mozer, F.S. Parallel electric fields in the upward current region of the aurora: Numerical solutions. Phys. Plasmas 2002, 9, 3685-3694. [CrossRef] 
32. Andersson, L.; Ergun, R.E.; Newman, D.L.; McFadden, J.P.; Carlson, C.W.; Su, Y.J. Characteristics of parallel electric fields in the downward current region of the aurora. Phys. Plasmas 2002, 9, 3600-3609. [CrossRef]

33. Cattell, C.; Neiman, C.; Dombeck, J.; Crumley, J.; Wygant, J.; Kletzing, C.A.; Peterson, W.K.; Mozer, F.S.; André, M. Large amplitude solitary waves in and near the Earth's magnetosphere, magnetopause and bow shock: Polar and cluster observations. Nonlinear Proc. Geophys. 2003, 10, 13-26. [CrossRef]

34. Schippers, P.; Blanc, M.; André, N.; Dandouras, I.; Lewis, G.R.; Gilbert, L.K.; Persoon, A.M.; Krupp, N.; Gurnett, D.A.; Coates, A.J.; et al. Multi-instrument analysis of electron populations in Saturn's magnetosphere. J. Geophys. Res. 2008, 113, A07208. [CrossRef]

35. Lynov, J.P.; Michelsen, P.; Pecseli, H.L.; Rasmussen, J.J.; Saeki, K.; Turikov, V.A. Observations of solitary structures in a magnetized, plasma loaded waveguide. Phys. Scr. 1979, 20, 328-335. [CrossRef]

36. Goldman, M.V.; Newman, D.L.; Ergun, R.E. Phase-space holes due to electron and ion beams accelerated by a current-driven potential ramp. Nonlinear Proc. Geophys. 2003, 10, 37-44. [CrossRef]

37. Jenab, S.M.H.; Brodin, G.; Juno, J.; Kourakis, I. Ultrafast electron holes in plasma phase space dynamics. Sci. Rep. 2021, 11, 16358. [CrossRef]

38. Simpson, J.A.; Bastian, T.S.; Chenette, D.L.; Lentz, G.A.; McKibben, R.B.; Pyle, K.R.; Tuzzolino, A.J. Saturnian trapped radiation and its absorption by satellites and rings: The first results from Pioneer 11. Science 1980, 207, 411-415. [CrossRef]

39. Verheest, F.; Hereman, W. Conservations laws and solitary wave solutions for gen-eralized Schamel equations. Phys. Scr. 1994, 50, 611-614. [CrossRef]

40. Williams, G.; Verheest, F.; Hellberg, M.A.; Anowar, A.G.M.; Kourakis, I. A Schamel equation for ion acoustic waves in superthermal plasmas. Phys. Plasmas 2014, 21,092103. [CrossRef]

41. Sultana, S.; Islam, S.; Mamun, A.A.; Schlickeiser, R. Oblique propagation of ion-acoustic solitary waves in a magnetized plasma with electrons following a generalized distribution function. Phys. Plasmas 2019, 26, 012107. [CrossRef]

42. Sultana, S.; Mannan, A.; Schlickeiser, R. Obliquely propagating electron-acoustic solitary waves in magnetized plasmas: The role of trapped superthermal electrons. Eur. Phys. J. D 2019, 73, 220. [CrossRef]

43. Mamun, A.A.; Cairns, R.A.; Shukla, P.K. Effects of vortex-like and non-thermal ion distributions on non-linear dust-acoustic waves. Phys. Plasmas 1996, 3, 2610-2614. [CrossRef]

44. Nejoh, Y.-N. The dust charging effect on electrostatic ion waves in a dusty plasma with trapped electrons. Phys. Plasmas 1997, 4, 2813-2819. [CrossRef]

45. Mamun, A.A. Nonlinear propagation of ion-acoustic waves in a hot magnetized plasma with vortexlike electron distribution. Phys. Plasmas 1998, 5, 322-324. [CrossRef]

46. Mamun, A.A.; Shukla, P.K. Electron-acoustic solitary waves via vortex electron distribution. J. Geophys. Res. Space Phys. 2002, 107, 1135. [CrossRef]

47. Mamun, A.A.; Shukla, P.K.; Stenflo, L. Obliquely propagating electron-acoustic solitary waves. Phys. Plasmas 2002, 9, 1474-1477. [CrossRef]

48. Tribeche, M.; Djebarni, L.; Schamel, H. Solitary ion-acoustic wave propagation in the presence of electron trapping and background nonextensivity. Phys. Lett. A 2012, 376, 3164-3174. [CrossRef]

49. Hafez, M.G.; Roy, N.C.; Talukder, M.R.; Ali, M.H.; Hafez, M.G.; Roy, N.C.; Talukder, M.R.; Ali, M.H. Effects of trapped electrons on the oblique propagation of ion acoustic solitary waves in elec-tron-positron-ion plasmas. Phys. Plasmas 2016, 23, 082904. [CrossRef]

50. Hassan, M.R.; Rajib, T.I.; Sultana, S. Electron-acoustic solitons in magnetized collisional nonthermal lasmas. arXiv 2019, arXiv:1912.04756.

51. Sultana, S.; Kourakis, I. Electron-scale electrostatic solitary waves and shocks: The role of superthermal electrons. Eur. Phys. J. D 2012, 66, 100. [CrossRef]

52. Ferdousi, M.; Sultana, S.; Mamun, A.A. Oblique propagation of ion-acoustic solitary waves in a magnetized electron-positron-ion plasma. Phys. Plasmas 2015, 22, 032117. [CrossRef]

53. Sultana, S.; Kourakis, I. Electron-scale dissipative electrostatic solitons in mul-ti-species plasmas. Phys. Plasmas 2015, 22, 102302. [CrossRef]

54. Sultana, S. Ion acoustic solitons in magnetized collisional non-thermal dusty plasmas. Phys. Lett. A 2018, 382, 1368-1373. [CrossRef]

55. Sultana, S.; Schlickeiser, R.; Elkamash, I.S.; Kourakis, I. Dissipative high-frequency envelope soliton modes in nonthermal plasmas. Phys. Rev. E 2018, 98, 033207. [CrossRef]

56. Jovanoviç, D.; Shukla, P.K.; Stenflo, L.; Pegoraro, F. Nonlinear model for electron phase-space holes in magnetized space plasmas. J. Geophys. Res. Space Phys. 2002, 107, 1110. [CrossRef]

57. Eliasson, B.; Shukla, P.K. Formation and dynamics of coherent structures involving phase-space vortices in plasmas. Phys. Rep. 2006, 422, 225-290. [CrossRef]

58. Schamel, H. Two-parametric, mathematically undisclosed solitary electron holes and their evolution equation. Plasma 2020, 3, 166-179. [CrossRef]

59. Schamel, H. Pattern formation in Vlasov-Poisson plasmas beyond Landau, as caused by the continuous spectra of electron and ion hole equilibria. arXiv 2021, arXiv:2110.01433. 